\title{
Two Unusual Presentation of Pulmonary Nocardiosis in Patients with Human Immunodeficiency Virus/Acquired Immunodeficiency Syndrome -A Case Report
}

\author{
Arivudainambi Veerappan Periasamy* \\ Department of pulmonary medicine, Mahatma Gandhi Memorial Government Hospital \& KAPV Government Medical College, India
}

Submission: September 18, 2017; Published: October 23, 2017

*Corresponding author: Arivudainambi Veerappan Periasamy, Senior assistant professor, Department of pulmonary medicine, Mahatma Gandhi Memorial Government Hospital \& KAPV Government Medical College, Trichy Tamilnadu, India, Pin-620017; Tel: 919443248165/919629599177; Email: arivudain@yahoo.com

Abstract

The present report highlights two cases of pulmonary nocardiosis diagnosed in Government KAPV Medical College Hospital, presenting as consolidation with multiple cavities, and fungal ball there by mimicking other common opportunistic pathogens. Early and correct microbiological diagnosis can help to decrease the disease-related morbidity and mortality. The development of complication like dissemination and resistance can be prevented by early diagnosis and treatment.

Keywords: Pulmonary nocardiosis; Immunocompromised host; Halo sign; Acid fast; Gram staining

\section{Introduction}

Nocardia, is rare in AIDS patients though an important opportunistic pathogen in many groups of immune compromised patients. Immunocompromise is a well-established risk factor for nocardiosis [1]. Nocardia can be considered as opportunistic pathogens, which can cause serious and disseminated disease in settings such as organ transplantation and lymphoreticular neoplasia, with the relative risk for progressive disease reflecting the level of immunesuppression. Timely diagnosis and appropriate treatment prevents dissemination and improves clinical outcomes.

The objectives of our case report are:

a) To discuss two unusual presentation of pulmonary Nocardiosis in patient with HIV/AIDS.

b) To make people recognize varied presentation of pulmonary nocardiosis.

c) To emphasize awareness of the possibility of Nocardia sp. as an opportunistic pathogen in AIDS patients.

\section{Case Presentation}

A 35 year old agricultural laborer was admitted with a fourweek history of shortness of breath and productive cough. Over the prior four months he also experienced generalized malaise, ten-kilogram weight loss, chills and fever.

He was started on Antituberculosis regimen in Tirupati in April 2014 and did not improve. He discontinued antituberculosis treatment after 4 months. He was HIV positive in a private lab on $05 / 12 / 2014$. He then came to our hospital on $7 / 12 / 2014$. He was not a Known diabetic nor a hypertensive. The patient denied smoking, alcohol and recreational drug use. Denied sick contacts and animal exposures, but admitted to unprotected heterosexual sexual exposure.

Physical findings were oxygen saturation 90\% room air, a respiratory rate of $26 /$ minute, temperature of $98.9^{\circ} \mathrm{F}$, Increased breath sounds, vocal fremitus and resonance, in the right lower lobes with tubular bronchial quality in the right infra axillary area.

He had a leukocytosis of $12,070 / \mathrm{mm} 3$ with granulocytes $71 \%$, lymphocytes $22 \%$ and LDH 360 units/ml. Renal and liver function tests were normal. HIV serology was positive and the absolute CD4 count was 30 .

Chest x-ray (Figure 1) showed initially airspace disease, accentuated in right lower lobes with air bronchogram. CT 
(Figure 2) scan taken it showed multiple bilateral consolidations with evidence of thin walled cavities in lower lobes posterior basal segments and middle lobe. Blood cultures were negative. The patient was empirically started on treatment doses of Trimethoprim/sulfamethoxazole for possible Pneumocystis jirovecii pneumonia pending bronchoscopic diagnosis.

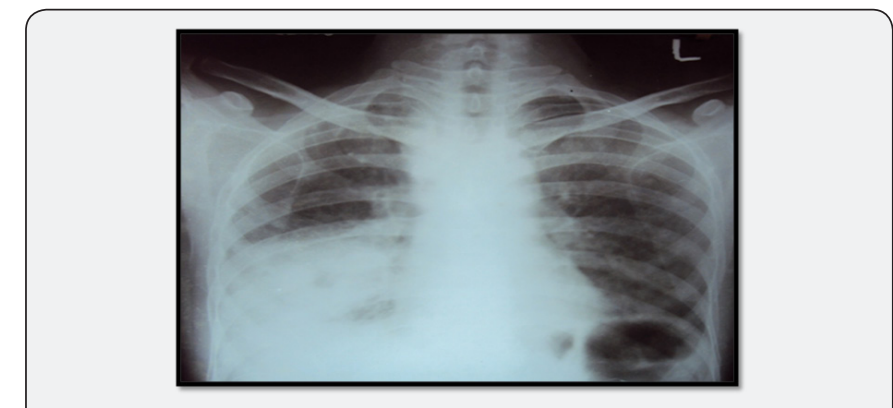

Figure 1: Chest $\mathrm{x}$-ray showing right lower lobeconsolidation with right paratracheal adenopathy.

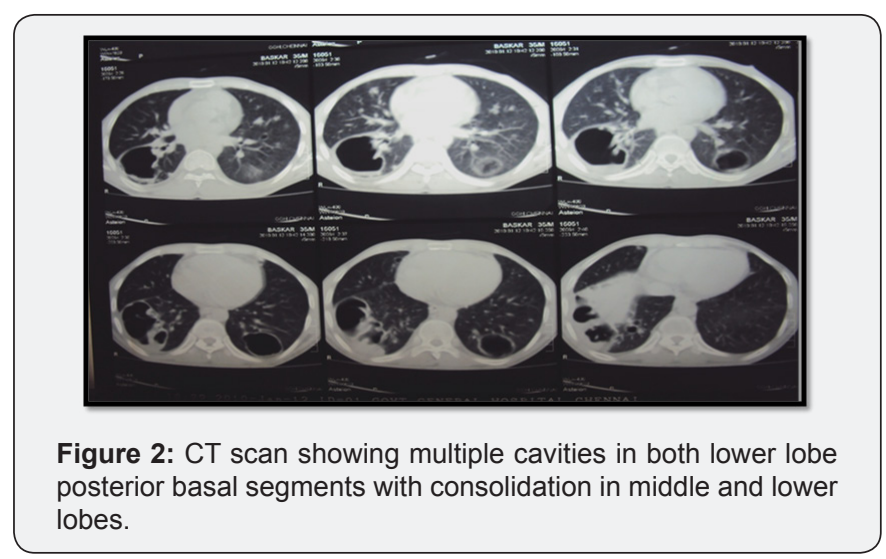

Expectorated sputa grew mixed flora and were positive on Gram staining for branching, beaded, filamentous bacteria and negative on stain for Pneumocystis and AFB but later positive for modified acid-fast staining. (Figure 3) The patient clinically improved on trimethoprim/sulfamethoxazole. bronchoscopic specimens were stain negative for AFB, Pneumocystis and viral cytologic changes and grew Nocardia species.

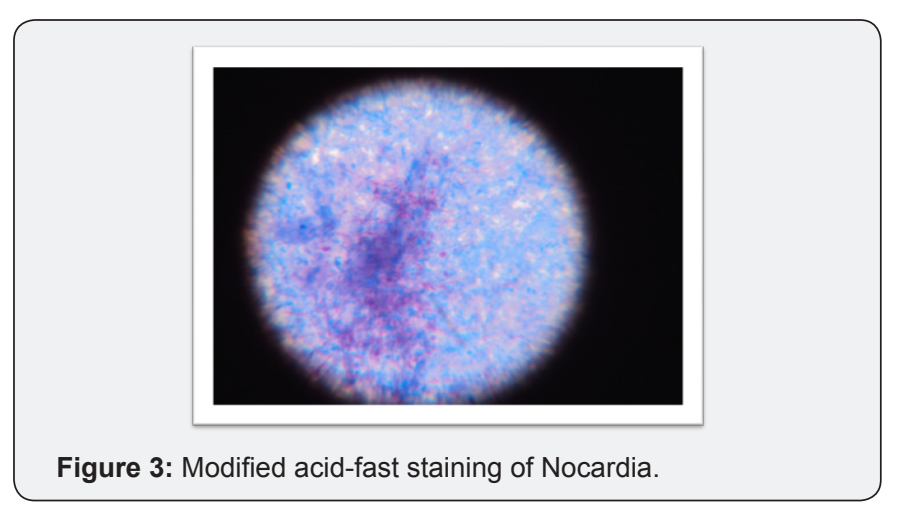

The patient was continued on high dose oral trimethoprim/ sulfamethoxazole and parenteral cefotaxime with continued clinical improvement and was discharged from the hospital on that regimen for outpatient follow-up. Subsequent chest X-rays showed improvement.

The second patient $30 y$ rs, female, house wife admitted to our hospital on June first, 2015 with the complaints of Cough with expectoration, breathlessness, Pain-left side of the chest, low grade fever for the past one month.

History include a diagnoses of HIV positivity on 24th May 2014 (Diagnosed at Erode). She was started on anti tuberculosis treatment in erode for her presenting Chest x-ray and clinical features. She was not a known diabetic, hypertensive, ischemic heart disease patient.

The patient denied smoking, alcohol and recreational drug use. Denied sick contacts and animal exposures, extra marital heterosexual sexual exposure but her husband died three years ago of undiagnosed illness.

Physical findings were oxygen saturation 90\% room air, a respiratory rate of $28 /$ minute, temperature of $99.9^{\circ} \mathrm{F}$, Increased breath sounds, vocal fremitus and resonance in the left upper and right upper lobes with tubular bronchial quality in the right mammary and infraclavicular areas. He had a leukocytosis of $15,400 / \mathrm{mm} 3$ with granulocytes $65 \%$, lymphocytes $22 \%$ and LDH 390 units $/ \mathrm{ml}$. Renal and liver function tests were normal. HIV serology was positive and the absolute CD4 count was 198.

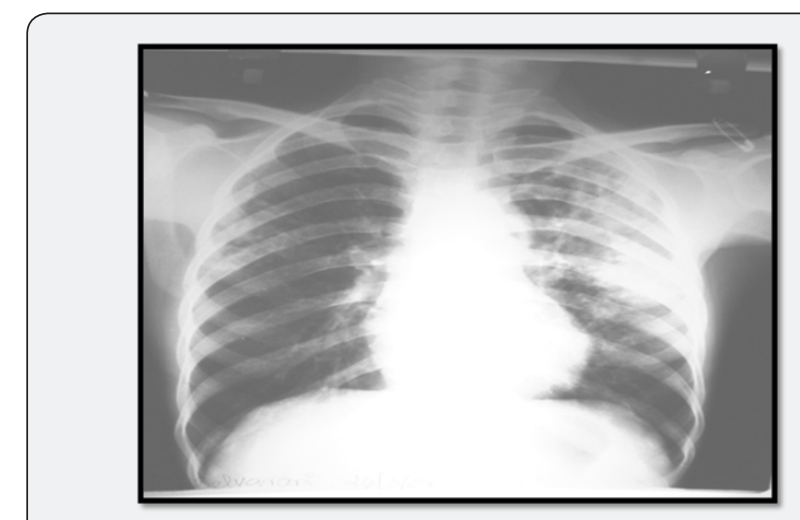

Figure 4: Chest X-ray showing ill-defined airspace opacity occupying the left upper and mid zones.

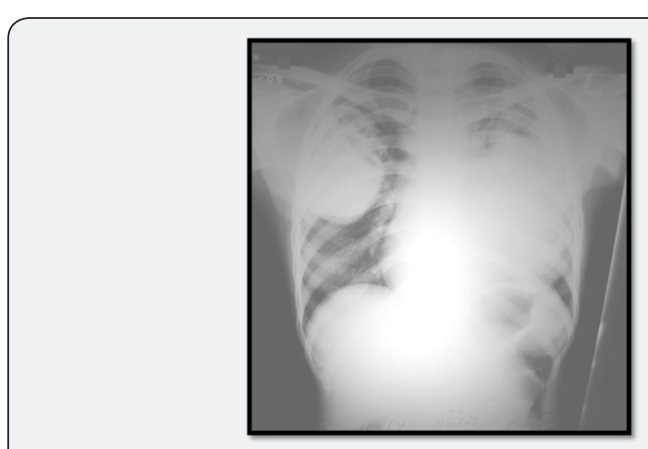

Figure 5: Showing halo sign in the right side. 
Chest x-ray (Figure 4) showed initially showed ill-defined air space opacity occupying the left upper and mid zones for which anti tuberculosis treatment started in Erode. Later X-ray (Figure 5) showed progression of consolidation in left side with appearance of well-defined oval mass like lesion with positive air crescent sign (Halo sign) in right upper zone.

Our differential diagnosis included tuberculosis, bacterial round pneumonias, fungal pneumonia especially aspergillus, mucor \& other infection like Nocardia. The patient was empirically started on treatment for possible Pneumocystis jirovecii pneumonia with doses of Trimethoprim/sulfamethoxazole pending investigation results.

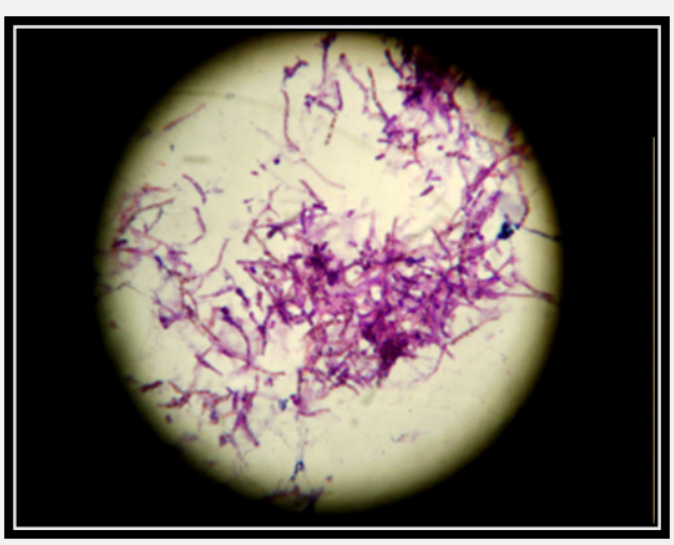

Figure 6: Gram staining shows gram positive branching beaded filamentous bacteria. Figure 5: Showing halo sign in the right side.

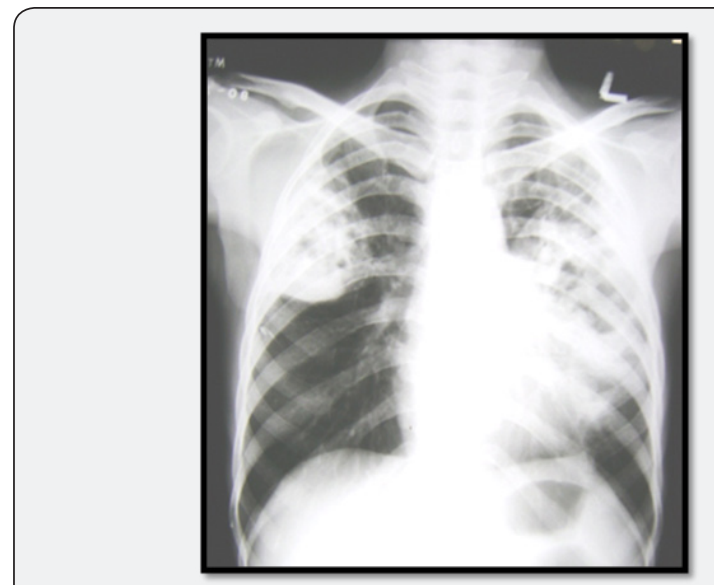

Figure 7: Chest $\mathrm{x}$-ray showing improvement taken after two weeks of treatment.

Expectorated sputa were positive on Gram staining for branching, beaded, filamentous bacteria (Figure 6) and negative on stain for Pneumocystis and AFB but later positive for modified acid-fast staining. The patient was continued on high dose oral Trimethoprim/sulfamethoxazole with continued clinical improvement and was discharged from the hospital on that regimen. Follow up X-ray showed improvement (Figure 7).

\section{Discussion}

Nocardia species are aerobic actinomycetes, opportunistic pathogen particularly in immunosuppressed patients. Due to incidental benefit of receiving trimethoprim/sulfamethoxazole prophylaxis for Pneumocystis pneumonia they are uncommon in AIDS patients. Primary nocardial infection includes pulmonary or cutaneous and/or subcutaneous lesions. Pulmonary disease is the predominant clinical presentation, occurring in more than $40 \%$ of reported cases [1]. [2] Pulmonary nocardiosis may be a fatal complication of advanced HIV infection and often presents as alveolar infiltrates that progress during therapy rather than as cavitary disease [3-5]. It occurs most commonly in severely immune compromised patients (CD4<200/mm3) [6]. Clinical manifestations of established infection include pneumonia, lung abscess, endobronchial inflammatory masses, and cavitary disease with contiguous extension to surface and deep structures, including empyema and effusion. Radiologic manifestations include irregular nodules (usually cavitating when large), reticulonodular or diffuse pneumonic infiltrates, and pleural effusions. The "halo sign," considered characteristic of aspergillosis in neutropenic patients has been described. Ninety percent of systemic cases are secondary to N. asteroides complex. Clinical pulmonary presentations may vary and includes acute or chronic pneumonia, lung abscess, or empyema. Disseminated infection is characterized by widespread abscess formation. The most commonly reported sites include the CNS and eyes (particularly the retina), skin and subcutaneous tissues, kidneys, joints, bone, and heart. Delay in the diagnosis and treatment of nocardia infections risks systemic dissemination and higher mortality. AIDS patients who present with undiagnosed pneumonias prior to starting Pneumocystis prophylaxis with trimethoprim, like our patient, may be at increased risk of having nocardia pulmonary infections as either the primary pulmonary process or as a co-infection with Pneumocystis, Mycobacterium tuberculosis or Mycobacterium avium-intracellulare. In the absence of a diagnosis made on expectorated or induced sputa, bronchoscopic specimens should be obtained for diagnosis and Nocardia infection considered in the differential diagnosis [7].

Clinical improvement is generally evident within 3 to 5 days or, at the most, 7 to 10 days after the initiation of appropriate therapy $[8,9]$. Parenteral therapy can usually be safely changed to an oral regimen after 3 to 6 weeks, depending on clinical response.

Recommendations on the duration of therapy are necessarily empirical and based primarily on reports of relapse after sulfonamide therapy of different durations $[6,9,10]$. There are rare case reports of cure of extra pulmonary abscesses after short-course parenteral therapy (7 to 8 weeks) with amikacin and surgical drainage [11], or amikacin plus ceftriaxone, as in a case of cerebral nocardiosis [12]. One- to 3-month courses of therapy are curative in patients with primary cutaneous infection, 
including sporotrichoid nocardiosis and superficial ulcers [6]. Prolonged therapy is required in patients with mycetoma [13]. Nonimmunosuppressed patients with pulmonary or systemic nocardiosis (excluding CNS involvement) should be treated for at least 6 months, and those with CNS involvement, for 12 months. These patients should be monitored for at least 1 year after completion of therapy to detect late relapses [14,15]. In HIV-negative, immunosuppressed patients, isolated pulmonary disease should be treated for at least 6 months and disseminated disease for 6 to 12 months, depending on the underlying state of immunosuppression and response to therapy. Therapy should be continued for 12 months or longer if there are intercurrent increases in immune suppression, for example, due to episodes of graft rejection.

\section{Conclusion}

We present cases of pulmonary nocardiosis with varied presentation like consolidation with cavitation (mimicking tuberculosis), fungal ball like lesion in HIV/AIDS. A reminder of this clinical entity and its potential clinical response to empiric trimethoprim/methoxazole therapy. Pulmonary nocardiosis may mimic Pulmonary TB [16]. HIV-TB-Nocardia Co infection may occur. High index of suspicion required to diagnose pulmonary Nocardiosis. Atypical manifestations may occur like the fungal ball. Pursuing a definitive diagnosis is critical to determine the appropriate course of antimicrobial therapy and improve patient survival.

\section{Acknowledgement}

Dr. Suriyakumar Madurai medical college.

\section{References}

1. Tamplin ML, McClung NM (1984) Quantitative studies of the relationship between trehalose lipids and virulence of Nocardia asteroids isolates. In: Ortiz-Ortiz L, Bojalil LF, Yakeloff V (Eds.) Biological, biochemical and biomedical aspects of actinomycetes. Fla: Academic Press Inc, Orlando, Florida, USA, pp. 251-258.

2. Peleg AY, Husain S, Qureshi ZA, Silveira FP, Sarumi M, et al. (2007) Risk factors, clinical characteristics, and outcome of Nocardia infection in organ transplant recipients: a matched case-control study. Clin Infect Dis 44(10): 1307-1314.
3. Beaman L, Beaman BL (1994) Nocardia species: host-parasite relationships. Clin Microbiol Rev 7(2): 213-264.

4. Kramer MR, Uttamchandani RB (1990) The radiographic appearance ofpulmonary nocardiosis associated with AIDS. Chest 98(2): 382-385.

5. Lucas SB, Hounnou A, Peacock C, Beaumel A, Kadio A, et al. (1994) Nocardiosis in HIV positive patients: an autopsy study in West Africa. Tuber Lung Dis 75(4): 301-307.

6. Uttamchandani RB, Daikos GL, Reyes RR, Fischl MA, Dickinson GM, et al. (1994) Nocardiosis in 30 patients with advanced Human Immunodeficiency Virus infection: clinical features and outcome. Clin Infect Dis 18(3): 348-353.

7. Javaly K, Horowitz HW, Wormser GP (1992) Nocardiosis in patients with human immunodeficiency virus infection: report of two cases and review of the literature. Medicine 71(3): 128-138.

8. McNeil MM, Brown JM, Hutwagner LC, Schiff TA (1995) Evaluation of therapy for Nocardia asteroides complex infections. Infect Dis Clin Pract 4(4): 287-292.

9. Wallace RJ, Septimus EJ, Williams TW, Conklin RH, Satterwhite TK, et al. (1982) Use of trimethoprim-sulfamethoxazole for treatment of infections due to Nocardia. Rev Infect Dis 4(2): 315-325.

10. Smego RA, Moeller MB, Gallis HA (1983) Trimethoprim sulfamethoxazole therapy for Nocardia infections. Arch Intern Med 143(4): 711-718.

11. Meier B, Metzger U, Müller F, Siegenthaler W, Lüthy R (1986) Successful treatment of apancreatic Nocardia asteroides abscess with amikacin and surgicaldrainage. J ClinMicrobiol 29(1): 150-151.

12. Garlando F, Bodmer T, Lee C, Zimmerli W, Pirovino M (1992) Successful treatment of disseminated nocardiosis complicated by cerebral abscess with ceftriaxone and amikacin: case report. Clin Infect Dis 15(6): 1039-1040.

13. Lopes CF (1996) Trimethoprim-sulfamethoxazole in the treatment ofactinomycotic mycetoma by Nocardia brasiliensis. Folha Medica 73: 89-92.

14. Geiseler PJ, Andersen BR (1979) Results of therapy in systemic nocardiosis. Am J Med Sci 278(3): 188-194.

15. Byrne E, Brophy BP, Perrett LV (1979) Nocardia cerebral abscess: new concepts in diagnosis management, and prognosis. J Neurol Neurosurg Psychiatry 42(11): 1038-1045.

16. Rasheed MU, Belay G (2008) Nocardiosis in HIV seropositive clinically suspected pulmonary tuberculosis patients. Trop Doct 38(1): 3435.

\section{Your next submission with Juniper Publishers will reach you the below assets}

- Quality Editorial service

- Swift Peer Review

- Reprints availability

- E-prints Service

- Manuscript Podcast for convenient understanding

- Global attainment for your research

- Manuscript accessibility in different formats

( Pdf, E-pub, Full Text, Audio)

- Unceasing customer service

Track the below URL for one-step submission https://juniperpublishers.com/online-submission.php 